\title{
Diagnostic retardé d'une rupture diaphragmatique traumatique
}

\section{Delayed diagnosis of a traumatic diaphragmatic rupture}

\section{P. Le Borgne $\cdot$ C.-E. Lavoignet $\cdot$ P. Bilbault}

Reçu le 12 octobre 2014; accepté le 17 décembre 2014

(C) SFMU et Lavoisier SAS 2015

Un homme de 37 ans s'est présenté aux urgences avec une douleur thoracique intermittente évoluant depuis plusieurs semaines. Le patient n'a aucun antécédent notable mais déclare avoir été victime six mois auparavant d'un accident de la voie publique à forte cinétique pour lequel le bilan lésionnel était négatif et l'imagerie normale. L'examen clinique initial retrouve une douleur en hypochondre gauche ainsi qu'une abolition du murmure vésiculaire homolatéral. L'ECG est normal, le patient bénéficie d'une radio de thorax (Fig. 1). Celle-ci retrouve une disparition de la coupole diaphragmatique gauche, une ascension des organes digestifs dans le thorax et une minime déviation médiastinale droite. Dix à $20 \%$ des lésions diaphragmatiques traumatiques passent initialement inaperçues. Dans la littérature, la rupture intéresse, dans plus de $80 \%$ des cas, la partie gauche du diaphragme, ce qui est infirmé par les études autopsiques retrouvant une répartition égale. Cette pathologie constitue un véritable challenge diagnostique pour le clinicien devant un examen clinique souvent aspécifique [1]. Il est alors important de réaliser une imagerie (même à distance du traumatisme) en cas de symptômes thoraco-abdominaux de novo afin de dépister ce type de lésion retardée. Ce patient a bénéficié en urgence d'une chirurgie diaphragmatique réparatrice par coelioscopie et est sortie sans séquelles.

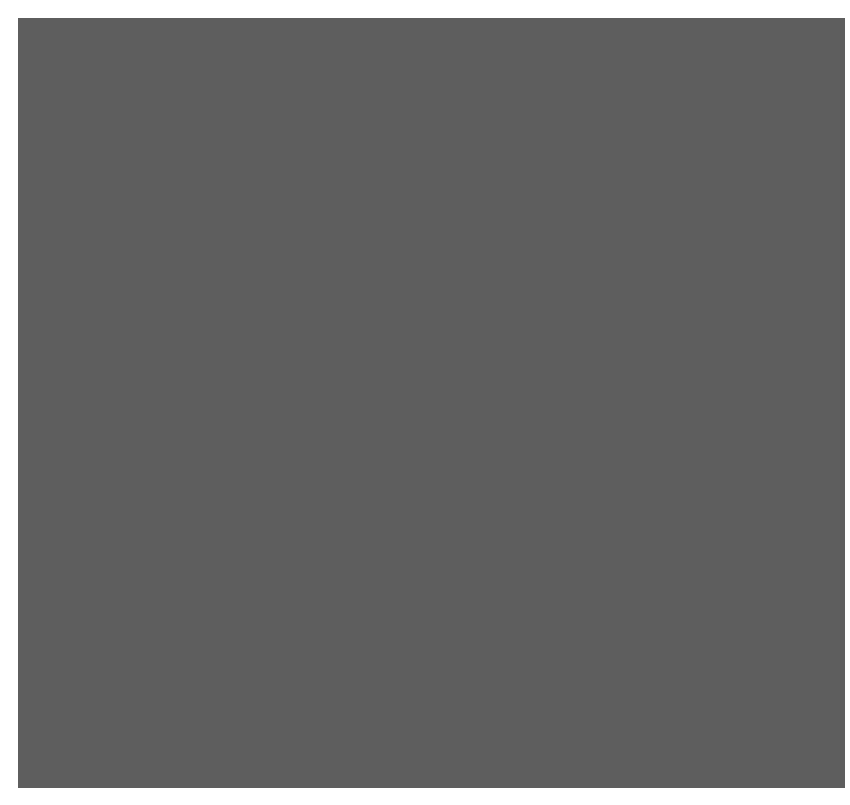

\section{Références}

1. Guth AA, Pachter HL, Kim U (1995) Pitfalls in the diagnosis of blunt diaphragmatic injury. Am J Surg 170:5-9

P. Le Borgne $(\bowtie) \cdot$ C.-E. Lavoignet $\cdot$ P. Bilbault Service d'accueil des urgences, CHU de Strasbourg, hôpital de Hautepierre, 1 avenue Molière,

F-67098 Strasbourg, France

e-mail : pierrick_med@yahoo.fr

P. Le Borgne $\cdot$ P. Bilbault

Faculté de médecine, université de Strasbourg,

4 rue Kirschleger, F-67000 Strasbourg 